within endoscopic reach, both $>2 \mathrm{~cm}$ in diameter $\&>5$ $\mathrm{cm}$ in length. There were no deaths due to $\mathrm{FB}$ ingestion.

Conclusions This is the largest study of Endoscopic FB removal from Western India. This study showed that the majority of the FB were ingested by children. Even those with injury to the bowel were asymptomatic. Since the size of FB did not determine it being within the reach of the endoscope and Groups S, NS \& C FBs are associated with injury to the bowel; we propose an attempt at removal should be made in all patients, whether symptomatic or not.

\section{IDDF2021-ABS-0160 CAUSES OF UPPER GI BLEEDING - EVALUATING THE AETIOLOGIES IN A 10- YEAR RETROSPECTIVE COHORT}

Teng Kiat Koh*, Jonathan Wei Jie Lee, Calvin Jianyi Koh. National University Hospital, Singapore

\subsection{6/gutjnl-2021-IDDF.163}

Background It is widely recognized that there are significant differences in the epidemiology of Upper Gastrointestinal Bleeding (UGIB) between Eastern and Western populations. We aim to provide an update of the prevalence of UGIB aetiologies in an Asian population in Singapore and to compare with recent data from a Western population.

Methods A retrospective, observational review of all patients who underwent oesophagogastroduodenoscopy for the indications of UGIB from 2011 to 2020 in the National University Hospital, Singapore, was analysed. We included cases performed for the following indications: haematemesis, coffee ground vomitus, melaena and bleeding gastrointestinal tract, The primary endoscopic findings were extracted and this provided a snapshot of aetiologies in the time period examined. The results were compared to that of other recent published prevalence data in other populations.

Results 5,691 cases were analysed. The three most common aetiologies of UGIB were peptic ulcer disease (PUD) (43.6\%), non-ulcer mucosal lesions (27.6\%) (gastritis, erosive gastritis, duodenitis, and erosive duodenitis) and oesophageal varices (8.03\%) (IDDF2021-ABS-0160 Figure 1. Aetiology of GI Bleeding 2011-2020).

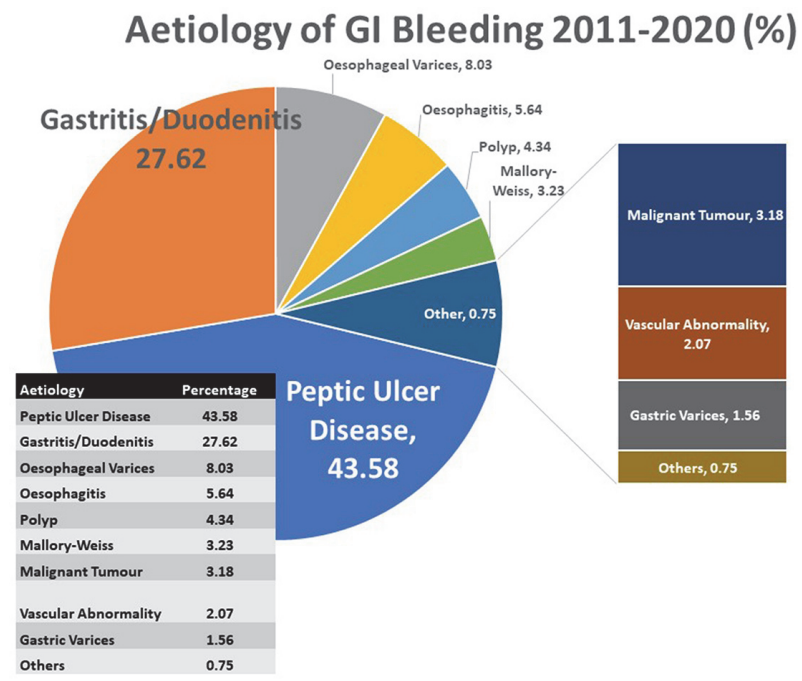

Abstract IDDF2021-ABS-0160 Figure 1
Comparing with a large representative US cohort (Wuerth 2017), although the overall prevalence of gastroduodenal ulcers/erosions was similar with PUD (47.1\%), there was more oesophagitis (15.2\% vs $5.6 \%)$ and less oesophageal varices $(1.8 \%$ vs $9.59 \%)(\mathrm{p}<0.001)$.

Conclusions These results provide an updated prevalence of GI bleeding aetiology in a large retrospective multi-ethnic Asian cohort. Of note, while the proportions of peptic ulcer disease and inflammatory conditions (erosive gastritis etc.) are similar between reported literature and the referenced US cohort, there is considerably more esophageal variceal bleeding and less esophagitis. These differences are likely due to underlying differences in disease patterns of the populations with Asia having more chronic viral hepatitis B and less reflux disease compared with western populations.

\section{IDDF2021-ABS-0171 LONG TERM OUTCOMES OF PALLIATIVE COLONIC STENT PLACEMENT IN MALIGNANT COLONIC OBSTRUCTION: EXPERIENCE FROM A TERTIARY CARE ONCOLOGY CENTER IN INDIA}

Sridhar Sundaram*, Raosaheb Rathod, Prachi Patil, Avanish Saklani, Shaesta Mehta. Tata Memorial Hospital, Mumbai, India

\subsection{6/gutjnl-2021-IDDF.164}

Background Colonic Self-expanding metal stent (SEMS) placement is the preferred method for palliation of malignant colonic obstruction. We analysed outcomes of patients who underwent colonic SEMS placement for palliation at a tertiary care oncology centre in Western India.

Methods A retrospective review of the endoscopy database was done for patients who underwent colonic SEMS placement at our center between January 2013 till December 2020. Demographic details, intent of stent placement, site of obstruction, length of stenosis, technical success of stenting, clinical success and complications (both immediate and long term) were noted.

Results 42 patients underwent colonic SEMS placement during the study period (Mean age 54.29 years, 50\% men). Obstruction was due to primary colonic malignancy in 32 (76.2\%) patients. Extra-colonic malignancies leading to obstruction included Gall Bladder cancer in 6 patients, Ovarian cancer in 3 patients and pancreatic cancer in 1 patient. Site of obstruction was sigmoid colon in 15 $(35.7 \%)$ patients, descending colon in 10 (23.8\%), rectum and rectosigmoid in 8 (19\%), distal right colon and hepatic flexure in $5(11.9 \%)$ and transverse colon in 4 (9.5\%). Metastatic disease was seen in $88.1 \%$. Peritoneal metastases were seen in $17(40.5 \%)$ patients. Median length of stricture was $5 \mathrm{~cm}$. Technical success was achieved in $100 \%$. Clinical success was achieved in $36(85.7 \%)$ patients. In 3 patients, there was a clinical failure of stent placement. In 3 patients, although there was partial relief of symptoms, recurrence of obstructive symptoms occurred in less than one week. There was no correlation between peritoneal carcinomatosis and clinical success $(p=0.700)$. Perforation during colonic SEMS placement occurred in 1 (2.4\%) patient. Stent migration was seen in 3 patients, needing surgery in 2 patients. Over a median follow-up of 8 months (0-21 months), stent block was seen in $6(14.2 \%)$ patients. Stent block developed after a median period of 6 months. Of 